\title{
SBP block: further considerations
}

\author{
Korean Journal of Anesthesiology Alessandro De Cassai and Christelle Correale \\ Department of Medicine - DIMED, Section of Anesthesiology and Intensive Care, University of Padua, Padua, Italy
}

We would like to thank Dr. Nair and Seelam [1] for the thoughtful comments on our technique.

Some important points have been highlighted that need further discussion and clarification.

The hypothesis behind our work was that erector spinae plane (ESP) block alone cannot provide adequate anesthetic coverage for breast surgery. The colleagues have suggested 30 $\mathrm{ml}$ as the optimal volume to avoid technique failure; however, a previous study [2] has reported that a volume of $25 \mathrm{ml}$ provides unsatisfactory anesthesia in a short stature patient. For this reason, we believe that further studies with a large population size are needed to have a final say on this topic.

We agree with the colleagues that long thoracic nerve, thoracodorsal nerve, and pectoral nerves are primarily motor nerves; however, we have to highlight that damage to these nerves could cause severe pain in the patients; in particular, a lesion to the long thoracic nerve causes a neuropathic pain in the scapular area [3]. A recent study comparing pectoral nerves block (Pecs block) to paravertebral block concluded that surgical patients receiving Pecs block had a significantly prolonged duration of postoperative analgesia with a lesser requirement for rescue analgesia, probably because of a more effective block of the brachial plexus [4]. Several mechanisms could play a role in the post-surgical development of pain: first, both proprioception and nociception have been described in these nerves; second, cervical and thoracic postganglionic fibers are carried by all the thoracic nerves; finally, a damage, spasm, or stretch of the mus-

Corresponding author: Alessandro De Cassai, M.D.

Department of Medicine - DIMED, Section of Anesthesiology and Intensive Care, University of Padua, 13, Gallucci Street, Padua 35121, Italy

Tel: +39-049-8213090, Fax: +39-049-8213090

Email: alessandro.decassai@gmail.com

ORCID: https://orcid.org/0000-0002-9773-1832

Received: January 29, 2019.

Revised: February 14, 2019.

Accepted: February 25, 2019. cles could result in myofascial pain, while the disruption and radiation of these nerves could play a role in the occurrence of neuropathic pain [5].

Secondarily, it could be important for the surgeon to work in a "motor blocked field" to avoid involuntary movements, especially when the patient is lightly sedated.

We want to make a last-but-not-the-least consideration regarding ESP block in patients with abnormalities in coagulation.

As stated by the American Society of Regional Anesthesia (ASRA) guidelines, deep perineuraxial techniques are discouraged because bleeding in a fixed, deep, non-compressible site could promote complications in suboptimal coagulation conditions; however, they conclude that the most serious complication of non-neuraxial regional technique is significant blood loss, and not neural deficits. Notably, almost all patients suffering complications underwent lumbar plexus block and no patient underwent an interfascial block.

Moreover, we believe that the unfortunate event of the formation of a neuraxial hematoma following an ESP block could happen for three reasons: a) ESP is a large plane where blood can flow freely from the cervical to the lumbar level; b) ESP is superficial, especially at the thoracic level, permitting an easy access for hematoma drainage; c) despite the clinical effect of ESP block that mimics a paravertebral block, the spread of fluid during ESP block to paravertebral space is still a matter of debate [5].

The ESP block does not seem to have the characteristics of a deep plexus block. In fact, it is rather superficial $(3-4 \mathrm{~cm}$ at the thoracic level), compressible, and not in a fixed space. For these reasons, we disagree with the colleagues in considering the ESP block a "deep plexus block."

Prospective studies regarding ESP block under suboptimal coagulation conditions are lacking, and although the preliminary reports are promising, we would like to spread the word of caution expressed by the colleagues over the administration of ESP block under altered coagulation conditions until more evidence is available.

Korean J Anesthesiol 2019 August 72(4): 392-393

https://doi.org/10.4097/kja.19036

(c) This is an open-access article distributed under the terms of the Creative Commons Attribution Non-Commercial License (http://creativecommons.org/ licenses/by-nc/4.0/), which permits unrestricted non-commercial use, distribution, and reproduction in any medium, provided the original work is properly cited. 


\section{Funding Statement}

Support was provided solely from institutional and/or departmental sources.

\section{Conflicts of Interest}

No potential conflict of interest relevant to this article was reported.

\section{Author Contributions}

Alessandro De Cassai (Conceptualization)

Christelle Correale (Conceptualization)

\section{ORCID}

Alessandro De Cassai, https://orcid.org/0000-0002-9773-1832

Christelle Correale, https://orcid.org/0000-0003-4222-3977

\section{References}

1. Nair AS, Seelam S. The risks associated with erector spinae plane block in patients with abnormalities of coagulation. Korean J Anesthesiol 2019; 72: 275-6.

2. Ueshima H, Otake H. Limitations of the Erector Spinae Plane (ESP) block for radical mastectomy. J Clin Anesth 2018; 51: 97.

3. Silva JB, Gerhardt S, Pacheco I. Syndrome of fascial incarceration of the long thoracic nerve: winged scapula. Rev Bras Ortop 2015; 50: 5737.

4. Kulhari S, Bharti N, Bala I, Arora S, Singh G. Efficacy of pectoral nerve block versus thoracic paravertebral block for postoperative analgesia after radical mastectomy: a randomized controlled trial. Br J Anaesth 2016; 117: 382-6.

5. Woodworth GE, Ivie RMJ, Nelson SM, Walker CM, Maniker RB. Perioperative breast analgesia: a qualitative review of anatomy and regional techniques. Reg Anesth Pain Med 2017; 42: 609-31. 